\title{
Totally nonsymplectic Anosov actions on tori and nilmanifolds
}

\author{
DAVID FISHER \\ BORIS KALININ \\ RALF SPATZIER
}

\begin{abstract}
We show that totally nonsymplectic Anosov actions of higher rank abelian groups on tori and nilmanifolds with semisimple linearization are $C^{\infty}$-conjugate to actions by affine automorphisms.
\end{abstract}

\section{Introduction}

Hyperbolic actions of abelian groups of rank at least 2 exhibit many surprising rigidity properties. Case in point is the local smooth rigidity of actions by automorphisms of tori and nilmanifolds and other algebraically defined actions. This means that perturbations of an action that are $C^{1}$-close for a finite set of generators are $C^{\infty}$-conjugate to the original action. It was established for algebraic actions with semisimple linear part by Katok and Spatzier in [18] and for some nonsemisimple actions on tori by Einsiedler and T Fisher in [4]. The higher rank situation is entirely different from the case of single Anosov diffeomorphisms and flows for which it is always easy to construct $C^{1}$-small perturbations which are not even $C^{1}$-conjugate.

Local smooth rigidity of algebraic actions gives strong support to the following conjecture by Katok and Spatzier.

Classification Conjecture All "irreducible" Anosov $\mathbb{Z}^{k}$ and $\mathbb{R}^{k}$-actions for $k \geq 2$ on any compact manifold are $C^{\infty}$-conjugate to algebraic actions.

Kalinin and Spatzier proved this conjecture for the special class of Cartan actions of abelian groups of rank at least 3 under some other more technical hypotheses [15]. Here we call an action Cartan if maximal nontrivial intersections of stable distributions of various elements, called coarse Lyapunov distributions, are one-dimensional and, together with the orbit, span the tangent space. Kalinin and Sadovskaya have results for more general Anosov actions of rank at least 2 where the condition on dimension 1 
is replaced by either uniform quasiconformality or a pinching condition on each coarse Lyapunov distribution $[13 ; 14]$. The basic idea of the proofs in all of these results is to build some smooth structures on various foliations and then combine them. However, this only works under strong assumptions on the action.

The general case of the conjecture remains out of reach. Thus it is natural to restrict attention to actions on tori and nilmanifolds where one usually refers to the conjecture as global rigidity. For these spaces, the classical results of Franks [6] and Manning [21] offer a different approach. Their work implies that any action $\alpha$ of an abelian group with at least one Anosov element on a torus or a nilmanifold is always $C^{0}$-conjugate to an action by affine Anosov automorphisms by some Hölder conjugacy $\phi$. Now to prove global rigidity it suffices to show smoothness of the conjugacy $\phi$. We call the latter action the linearization of $\alpha$ and refer to Section 2 for a precise definition. On the torus the linearization is essentially given by the induced action on homology. Note that in the nilmanifold case, the term "linearization" is a bit of a misnomer as the action by automorphisms is not really linear.

The idea that a $C^{0}$ conjugacy can be used to get $C^{\infty}$-rigidity appears already in Hurder's work on deformation rigidity of lattice actions on tori [10] and later in Katok and Lewis [17] for both their local and global rigidity theorems for Cartan actions on tori. It also formed the basis of the argument for local rigidity by Katok and Spatzier [18]. In the different context of local rigidity of algebraic actions of lattices in higher rank groups, work of Katok and Spatzier [18] and later Fisher and Margulis [5] and Margulis and Qian [22] also involves finding a $C^{0}$ conjugacy that is improved to $C^{\infty}$ using the presence of higher rank abelian subgroups in the acting group. Rodriguez Hertz established global rigidity for $\mathbb{Z}^{k}$ actions on tori with at least one Anosov element whose linearization has coarse Lyapunov foliations of dimensions one or two and either has a maximal rank or satisfies additional bunching assumptions on the full (un)stable distribution of the Anosov element [25]. To date, all results require that the derivatives of either the action or its linearization along the coarse Lyapunov distributions satisfy a pinching assumption. This means that the ratio of maximal over minimal contraction is controlled, eg less than 2. In this paper, we overcome this problem for the first time by a combination of the use of nonstationary normal forms and holonomy arguments. Beyond achieving a superior result, the use of the two tools is also completely novel. We use limits of holonomy maps to define homogeneous structures on certain foliations. This has never been done before. Furthermore, we make use of measurable normal forms for the first time in the context of global rigidity of actions. Previously measurable normal forms have only been used to study invariant measures.

Continuous normal forms were already introduced for the proof of local rigidity in [18]. In essence they give coordinate charts in which the derivatives of the map along 
contracting foliations take values in a finite dimensional Lie group. Moreover, the dependence of the coordinates on the base point is continuous in the $C^{\infty}$-topology. Existence of continuous normal forms is guaranteed if the derivatives of the maps under consideration satisfy a spectral gap condition along the given contracting foliation. While such spectral gaps are automatic for $C^{1}$-perturbations of algebraic systems and also for one dimensional foliations, they fail to hold in general. In particular we cannot assume such spectral gaps for the proof of global rigidity. Instead, we use a measurable version of the nonstationary normal forms theory where the "measurable" spectral gap condition is always satisfied by Oseledec' Multiplicative Ergodic Theorem.

Let us next summarize some elements from the structure theory of higher rank abelian actions; see Section 2 for more details. They preserve a probability measure of full support. One can find a common Lyapunov splitting of the tangent bundle $T M=\bigoplus_{i} E_{i}$ which refines the Lyapunov splittings of each individual element. Moreover, if $v \in E_{i}$, the Lyapunov exponent of $v$ defines a linear functional, the Lyapunov functional, on the acting $\mathbb{Z}^{k}$ which we think of as a linear functional on the ambient $\mathbb{R}^{k}$. For actions by affine automorphisms the Lyapunov exponents are nothing but the logarithms of the absolute values of the eigenvalues of the automorphisms. A Weyl chamber is a connected component of $\mathbb{R}^{k}$ minus all the hyperplane kernels of the Lyapunov functionals. We will need to make the assumption that every Weyl chamber defined by the linearization contains an Anosov element in the nonlinear action. As we will later see that the Weyl chambers on the two sides agree, we abbreviate this by saying that every Weyl chamber contains an Anosov element. This allows us to define the coarse Lyapunov foliations as the maximal intersections of stable foliations of Anosov elements. Hence these foliations are Hölder with smooth leaves.

Recall that a matrix is semisimple if it is diagonalizable over $\mathbb{C}$. We call an action by affine automorphisms of a nilmanifold semisimple if the linear part of every element acts by a semisimple matrix.

Finally, we call a $\mathbb{Z}^{k}$-action TNS or totally nonsymplectic if any two $v \in E_{i}$ and $w \in E_{j}$ belong to the stable distribution of some element $a \in \mathbb{Z}^{k}$. This excludes the possibility of a bilinear form invariant under the action, hence the name.

The main result of this paper proves global rigidity for totally nonsymplectic actions. Recall that a nilmanifold is a quotient $N / \Gamma$ where $N$ is a simply connected nilpotent Lie group and $\Gamma$ is a cocompact discrete subgroup.

Theorem 1.1 Suppose $\alpha$ is a $C^{\infty}$-action of $\mathbb{Z}^{k}, k \geq 2$, on a nilmanifold $N / \Gamma$. Assume the linearization $\rho$ of $\alpha$ is semisimple and TNS and there is an Anosov element in each Weyl chamber of $\alpha$. Then $\alpha$ is $C^{\infty}$-conjugate to $\rho$. 
As discussed above, this theorem is the first that does not require pinching conditions. Moreover, it also yields the first global rigidity result for Anosov actions on nilmanifolds which are not tori. Indeed, in all earlier results the pinching condition, together with various additional assumptions such as integrability or absence of certain resonances, forced the nilmanifold to be a torus.

Call a linear $\mathbb{Z}^{k}$ action on a torus totally reducible if every rational invariant torus has a rational invariant complement. There is a similar though more complicated notion for nilmanifolds which we describe below in Section 9. We will show that total reducibility is equivalent to semisimplicity, and thus we immediately get the next result:

Corollary 1.2 Suppose $\alpha$ is a $C^{\infty}$-action of $\mathbb{Z}^{k}, k \geq 2$, on a nilmanifold $N / \Gamma$. Assume the linearization $\rho$ of $\alpha$ is totally reducible and TNS and there is an Anosov element in each Weyl chamber of $\alpha$. Then $\alpha$ is $C^{\infty}$-conjugate to $\rho$.

To prove the corollary from the theorem, we prove that any totally reducible action is semisimple.

Our results have some applications to global rigidity for actions of higher rank lattices. Margulis and Qian prove that any Anosov action of a higher rank lattice $\Gamma$ on a nilmanifold with a common fixed point for the entire group action is continuously conjugate to an action by affine automorphisms [22]. It is well known that such $\Gamma$ contains many abelian subgroups isomorphic to $\mathbb{Z}^{k}$, where $k$ is the real rank of $\Gamma$, and that the Anosov $\Gamma$ action restricts to an Anosov $\mathbb{Z}^{k}$ action. If some $\mathbb{Z}^{k}$ subgroup satisfies the conditions of Theorem 1.1, it then follows from our results that the conjugacy is smooth, and therefore that the full $\Gamma$ action is smoothly conjugate to an action by affine automorphisms.

Let us briefly indicate the main elements in the proof of Theorem 1.1. As discussed above we show that the topological conjugacy $\phi$ is smooth. For this, we first suspend the $\mathbb{Z}^{k}$-action to an $\mathbb{R}^{k}$-action. Then we fix a coarse Lyapunov foliation and for almost every leaf we construct a transitive group of smooth transformations which is intertwined by $\phi$ with the group of translations of the corresponding leaf for the linearization. As in other proofs of rigidity theorems eg in [18], we use limits of return maps. Unlike earlier proofs however, we do not directly use the acting group but rather holonomies along transversal coarse Lyapunov foliations. First we show that these holonomies are smooth. For this we establish existence of elements which contract the fixed coarse Lyapunov foliation slower than a transversal one. Then we show that the holonomies centralize suitable elements of $\mathbb{R}^{k}$ and hence preserve measurable nonstationary normal forms. It follows that limits of such holonomies are still smooth and define the desired transitive group actions. Once the smoothness of $\phi$ is established 
for a.e. leaf of each coarse Lyapunov foliation, the smoothness of holonomies gives the global smoothness of $\phi$. A more detailed outline of the proof is given in Section 3, after all relevant notions have been defined.

Acknowledgements We would like to thank K Burns, D Dolgopyat, F Ledrappier, Y Pesin, $\mathrm{J}$ Rauch and A Wilkinson for a number of discussions on subjects related to this paper.

The first author was supported in part by NSF grant DMS-0643546. The second author was supported in part by NSF grant DMS-0701292. The third author was supported in part by NSF grants DMS-0906085 and DMS-0604857.

\section{Preliminaries}

Throughout the paper, the smoothness of diffeomorphisms, actions, and manifolds is assumed to be $C^{\infty}$, even though all definitions and some of the results can be formulated in lower regularity.

\subsection{Anosov actions of $\mathbb{Z}^{k}$ and $\mathbb{R}^{k}$}

Let $a$ be a diffeomorphism of a compact manifold $M$. We recall that $a$ is Anosov if there exist a continuous $a$-invariant decomposition of the tangent bundle $T M=E_{a}^{s} \oplus E_{a}^{u}$ and constants $K>0, \lambda>0$ such that for all $n \in \mathbb{N}$,

$$
\begin{array}{rlrl}
\left\|D a^{n}(v)\right\| & \leq K e^{-\lambda n}\|v\| & & \text { for all } v \in E_{a}^{s}, \\
\left\|D a^{-n}(v)\right\| \leq K e^{-\lambda n}\|v\| & & \text { for all } v \in E_{a}^{u} .
\end{array}
$$

The distributions $E_{a}^{s}$ and $E_{a}^{u}$ are called the stable and unstable distributions of $a$.

Now we consider a $\mathbb{Z}^{k}$ action $\alpha$ on a compact manifold $M$ via diffeomorphisms. The action is called Anosov if there is an element which acts as an Anosov diffeomorphism. For an element $a$ of the acting group we denote the corresponding diffeomorphisms by $\alpha(a)$ or simply by $a$ if the action is fixed.

For a $\mathbb{Z}^{k}$ action $\alpha$ on a manifold $M$, there is an associated $\mathbb{R}^{k}$ action $\tilde{\alpha}$ on a manifold $\mathcal{S}$ given by the standard suspension construction in Kalinin and Katok [11]. Briefly, this is the action of $\mathbb{R}^{k}$ by left translations on $\left(\mathbb{R}^{k} \times M\right) / \mathbb{Z}^{k}$. Here $\left(\mathbb{R}^{k} \times M\right) / \mathbb{Z}^{k}$ is the quotient of $\mathbb{R}^{k} \times M$ by the $\mathbb{Z}^{k}$-action on $\mathbb{R}^{k} \times M$ given by $z(r, p)=(r-z, z(p))$. We will refer to $\tilde{\alpha}$ as the suspension of $\alpha$. It generalizes the suspension flow of a diffeomorphism. Similarly, the manifold $\mathcal{S}$ is a fibration over the "time" torus $\mathbb{T}^{k}$ with fiber $M$. 
Definition 2.1 Let $\alpha$ be a smooth action of $\mathbb{R}^{k}$ on a compact manifold $M$. An element $a \in \mathbb{R}^{k}$ is called Anosov or normally hyperbolic for $\alpha$ if there exist positive constants $\lambda, K$ and a continuous $\alpha$-invariant splitting of the tangent bundle

$$
T M=E_{a}^{s} \oplus E_{a}^{u} \oplus T \mathcal{O}
$$

where $T \mathcal{O}$ is the tangent distribution of the $\mathbb{R}^{k}$-orbits, and (1) holds for all $n \in \mathbb{N}$.

An $\mathbb{R}^{k}$ action is called Anosov if some element $a \in \mathbb{R}^{k}$ is Anosov. Note that $a \in \mathbb{Z}^{k}$ is Anosov for $\alpha$ if and only if it is Anosov for $\widetilde{\alpha}$. Thus if $\alpha$ is an Anosov $\mathbb{Z}^{k}$ action then $\tilde{\alpha}$ is an Anosov $\mathbb{R}^{k}$ action.

Both in the discrete and the continuous case it is well-known that the distributions $E_{a}^{s}$ and $E_{a}^{u}$ are Hölder continuous and tangent to the stable and unstable foliations $\mathcal{W}_{a}^{s}$ and $\mathcal{W}_{a}^{u}$ respectively; see Hirsch, Pugh and Shub [9]. The leaves of these foliations are $C^{\infty}$ injectively immersed Euclidean spaces. Locally, the immersions vary continuously in the $C^{\infty}$ topology. In general, the distributions $E^{s}$ and $E^{u}$ are only Hölder continuous transversally to the corresponding foliations.

\subsection{Lyapunov exponents and coarse Lyapunov distributions}

First we recall some basic facts from the theory of nonuniform hyperbolicity for a single diffeomorphism; see for example Barreira and Pesin [2]. Then we consider $\mathbb{Z}^{k}$ and $\mathbb{R}^{k}$ actions concentrating on the continuous time case on the case, we refer to Kalinin and Spatzier [15] and Kalinin and Sadovskaya [13] for more details.

Let $a$ be a diffeomorphism of a compact manifold $M$ preserving an ergodic probability measure $\mu$. By Oseledec' Multiplicative Ergodic Theorem, there exist finitely many numbers $\chi_{i}$ and an invariant measurable splitting of the tangent bundle $T M=\bigoplus E_{i}$ on a set of full measure such that the forward and backward Lyapunov exponents of $v \in E_{i}$ are $\chi_{i}$. This splitting is called Lyapunov decomposition. We define the stable distribution of $a$ with respect to $\mu$ as $E_{a}^{-}=\bigoplus_{\chi_{i}<0} E_{i}$. The subspace $E_{a}^{-}(x)$ is tangent $\mu$-a.e. to the stable manifold $W_{a}^{-}(x)$. More generally, given any $\theta<0$ we can define the strong stable distribution by $E_{a}^{\theta}=\bigoplus_{\chi_{i} \leq \theta} E_{i}$ which is tangent $\mu$-a.e. to the strong stable manifold $W_{a}^{\theta}(x) . W_{a}^{\theta}(x)$ is a smoothly immersed Euclidean space. For a sufficiently small ball $B(x)$, the connected component of $W_{a}^{\theta}(x) \cap B(x)$, called local manifold, can be characterized by the exponential contraction property

$$
W_{a}^{\theta, \text { loc }}(x)=\left\{y \in B(x) \mid \operatorname{dist}\left(a^{n} x, a^{n} y\right) \leq C e^{(\theta+\varepsilon) n} \forall n \in \mathbb{N}\right\} .
$$

The unstable distributions and manifolds are defined similarly. In general, $E_{a}^{-}$is only measurable and depends on the measure $\mu$. However, if $a$ is an Anosov diffeomorphism, 
or an Anosov element of an $\mathbb{R}^{k}$ action, then $E_{a}^{-}$for any measure always agrees with the continuous stable distribution $E_{a}^{s}$. Indeed, $E_{a}^{s}$ cannot contain a vector with a nontrivial component in some $E_{j}$ with $\chi_{j} \geq 0$ since such a vector does not satisfy (1). Hence $E_{a}^{s} \subset \bigoplus_{\chi_{i}<0} E_{i}$. Similarly, the unstable distribution $E_{a}^{u} \subset \bigoplus_{\chi_{i}>0} E_{i}$. Since $E_{a}^{s} \oplus E_{a}^{u}$ is transverse to the orbit, both inclusions have to be equalities.

Let $\mu$ be an ergodic probability measure for an $\mathbb{R}^{k}$ action $\alpha$ on a compact manifold $M$. By commutativity, the Lyapunov decompositions for individual elements of $\mathbb{R}^{k}$ can be refined to a joint invariant splitting for the action. The following proposition from [15] describes the Multiplicative Ergodic Theorem for this case. See [13] for the discrete time version and [11] for more details on the Multiplicative Ergodic Theorem and related notions for higher rank abelian actions.

Proposition 2.2 Let $\alpha$ be a smooth action of $\mathbb{R}^{k}$ and let $\mu$ be an ergodic invariant measure. There are finitely many linear functionals $\chi$ on $\mathbb{R}^{k}$, a set of full measure $\mathcal{P}$, and an $\alpha$-invariant measurable splitting of the tangent bundle $T M=T \mathcal{O} \oplus \oplus E_{\chi}$ over $\mathcal{P}$, where $\mathcal{O}$ is the orbit foliation, such that for all $a \in \mathbb{R}^{k}$ and $v \in E_{\chi}$, the Lyapunov exponent of $v$ is $\chi(a)$, ie

$$
\lim _{t \rightarrow \pm \infty} t^{-1} \log \|(D \alpha(t a))(v)\|=\chi(a),
$$

where $\|\cdot\|$ is a continuous norm on $T M$.

The splitting $\bigoplus E_{\chi}$ is called the Lyapunov decomposition, and the linear functionals $\chi$ are called the Lyapunov exponents of $\alpha$. The hyperplanes $\operatorname{ker} \chi \subset \mathbb{R}^{k}$ are called the Lyapunov hyperplanes or Weyl chamber walls, and the connected components of $\mathbb{R}^{k}-\bigcup_{\chi}$ ker $\chi$ are called the Weyl chambers of $\alpha$. The elements in the union of the Lyapunov hyperplanes are called singular, and the elements in the union of the Weyl chambers are called regular. We note that the corresponding notions for a $\mathbb{Z}^{k}$ action and for its suspension are directly related. In particular, the nontrivial Lyapunov exponents are the same. In addition, for the suspension there is one identically zero Lyapunov exponent corresponding to the orbit distribution. From now on, the term Lyapunov exponent will always refer to the nonzero functionals.

Consider a $\mathbb{Z}^{k}$ action by automorphisms of a torus or a nilmanifold $M=N / \Gamma$. In this case, the Lyapunov decomposition is determined by the eigenspaces of the automorphisms, and the Lyapunov exponents are the logarithms of the moduli of the eigenvalues. Hence they are independent of the invariant measure, and they give uniform estimates of expansion and contraction rates. Also, every Lyapunov distribution is smooth and integrable. 
In the nonalgebraic case, the individual Lyapunov distributions are in general only measurable and depend on the given measure. This can be already seen for a single diffeomorphism, even if Anosov. However, as we observed above, the full stable distribution $E_{a}^{s}$ of an Anosov element $a$ always agrees with $\bigoplus_{\chi(a)<0} E_{\chi}$ on a set of full measure for any measure.

For higher rank actions, coarse Lyapunov distributions play a similar role to the stable and unstable distributions for an Anosov diffeomorphism. For any Lyapunov functional $\chi$ the coarse Lyapunov distribution is the direct sum of all Lyapunov spaces with Lyapunov functionals positively proportional to $\chi$ :

$$
E^{\chi}=\bigoplus E_{\chi^{\prime}}, \quad \chi^{\prime}=c \chi \text { with } c>0 .
$$

For an algebraic action such a distribution is a finest nontrivial intersection of the stable distributions of certain Anosov elements of the action. For nonalgebraic actions, however, it is not a priori clear. It was shown in [15, Proposition 2.4] that, in the presence of sufficiently many Anosov elements, the coarse Lyapunov distributions are welldefined, continuous, and tangent to foliations with smooth leaves (see Proposition 2.2 in [14] for the discrete time case). We denote the set of all Anosov elements in $\mathbb{Z}^{k}$ or $\mathbb{R}^{k}$ by $\mathcal{A}$.

Proposition 2.3 Let $\alpha$ be an Anosov action of $\mathbb{Z}^{k}$ or $\mathbb{R}^{k}$ and let $\mu$ be an ergodic probability measure for $\alpha$ with full support. Suppose that there exists an Anosov element in every Weyl chamber defined by $\mu$. Then for each Lyapunov exponent $\chi$ the coarse Lyapunov distribution can be defined as

$$
E^{\chi}(p)=\bigcap_{\{a \in \mathcal{A} \mid \chi(a)<0\}} E_{a}^{s}(p)=\bigoplus_{\left\{\chi^{\prime}=c \chi \mid c>0\right\}} E_{\chi^{\prime}}(p)
$$

on the set $\mathcal{P}$ of full measure where the Lyapunov exponents exist. Moreover, $E^{\chi}$ is Hölder continuous, and thus it can be extended to a Hölder distribution tangent to the foliation $\mathcal{W}^{\chi}=\bigcap_{\{a \in \mathcal{A} \mid \chi(a)<0\}} \mathcal{W}_{a}^{s}$ with uniformly $C^{\infty}$ leaves.

Note that ergodic measures with full support always exist if a $\mathbb{Z}^{k}$ action contains a transitive Anosov element.

A natural example is given by the measure $\mu$ of maximal entropy for such an element, which is unique [16, Corollary 20.1.4] and hence is invariant under the whole action.

Since a coarse Lyapunov distribution is defined by a collection of positively proportional Lyapunov exponents it can be uniquely identified with the corresponding positive (negative) set of these functionals, called the positive (negative) Lyapunov half-space, or with the oriented Lyapunov hyperplane that separates them. 
The action is called totally nonsymplectic, or TNS, if there are no negatively proportional Lyapunov exponents. Equivalently, any two different negative Lyapunov half-spaces have nontrivial intersection. Therefore, any pair of coarse Lyapunov distributions for such an action is contracted by the elements in this intersection.

\section{3 $\mathbb{Z}^{k}$ and $\mathbb{R}^{k}$ actions on tori and nilmanifolds}

Let $f$ be an Anosov diffeomorphism of a torus or, more generally, a nilmanifold $M=N / \Gamma$, where $N$ is a simply connected nilpotent Lie group and $\Gamma$ is a cocompact lattice. By the results of Franks [6] and Manning [21], $f$ is topologically conjugate to an Anosov automorphism $A: M \rightarrow M$, ie there exists a homeomorphism $\phi: M \rightarrow M$ such that $A \circ \phi=\phi \circ f$. The conjugacy $\phi$ is bi-Hölder, ie both $\phi$ and $\phi^{-1}$ are Hölder continuous with some Hölder exponent $\gamma$.

Now we consider an Anosov $\mathbb{Z}^{k}$ action $\alpha$ on a nilmanifold $M$. Fix an Anosov element $a$ for $\alpha$. Then we have $\phi$ which conjugates $\alpha(a)$ to an automorphism $A$. By [27, Corollary 1] any homeomorphism of $M$ commuting with $A$ is an affine automorphism. Hence we conclude that $\phi$ conjugates $\alpha$ to an action $\rho$ by affine automorphisms. We will call $\rho$ an algebraic action and refer to it as the linearization of $\alpha$.

Now we describe the preferred invariant measure for $\alpha$ ( $\operatorname{cf}[12$, Remark 1]). We denote by $\lambda$ the normalized Haar measure on the nilmanifold $M$. Note that $\lambda$ is invariant under any affine automorphism of $M$ and is the unique measure of maximal entropy for any affine Anosov automorphism.

Proposition 2.4 The action $\alpha$ preserves an absolutely continuous measure $\mu$ with smooth positive density. Moreover, $\mu=\phi_{*}^{-1}(\lambda)$ and for any Anosov element $a \in \mathbb{Z}^{k}$, $\mu$ is the unique measure of maximal entropy for $\alpha(a)$.

Proof Let $J$ denote the Jacobian of $\alpha$ with respect to the Haar measure $\lambda$, more precisely, for any $b \in \mathbb{Z}^{k}$ the function $J_{b}(x)$ denotes the density of the push forward measure $\alpha(b)_{*}(\lambda)$ with respect to $\lambda$. Since the conjugacy $\phi$ is bi-Hölder, $\log \left(J \circ \phi^{-1}\right)$ is a Hölder cocycle over the linearization $\rho$. By rigidity of Hölder cocycles for irreducible algebraic $\mathbb{Z}^{k}$-actions [18], this cocycle is Hölder cohomologous to a constant one, ie there exists a linear functional $c: \mathbb{Z}^{k} \rightarrow \mathbb{R}$ and a Hölder continuous function $\Phi$ on $M$ such that for all $b \in \mathbb{Z}^{k}$ and $x \in M$,

$$
\log \left(J_{b} \circ \phi^{-1}(x)\right)=c(b)+\Phi(\rho(b) x)-\Phi(x) .
$$

a constant $c$. Hence for the function $\Psi=\Phi \circ \phi$ we have

$$
\log \left(J_{b}(x)\right)=c(b)+\Psi(\alpha(b) x)-\Psi(x) .
$$


Let $\mu$ be the measure $e^{-\Psi(x)} \lambda$ normalized by $\mu(M)=1$. It follows that the Jacobian $\tilde{J}$ of $\alpha$ with respect to $\mu$ is constant, $\widetilde{J}_{b}(x)=e^{c(b)}$. Since $\alpha(b)$ is a diffeomorphisms we have $\int_{M} \widetilde{J}_{b}(x) d \mu=1$ and hence $e^{c(b)}=1$. Thus $\alpha$ preserves $\mu$.

Let $a$ be an Anosov element of $\alpha$. Then the density of its absolutely continuous measure $\mu$ is $C^{1}$ [16, Theorem 19.2.7], and in fact $C^{\infty}$ [20, Corollary 2.1]. Also, $\mu$ is the equilibrium state of $\log \left(J^{u}\right)$ [16, Theorem 20.4.1], where $J^{u}$ is the Jacobian of $\alpha(a)$ along its unstable distribution. As before, we have that $\log \left(J^{u}\right)$ is cohomologous to a constant function. Since the measure of maximal entropy of the transitive Anosov diffeomorphism $\alpha(a)$ is the equilibrium state of the constant function [16, Theorem 20.1.3], and since the equilibrium state is unique [16, Theorem 20.3.7] we conclude that $\mu$ is the measure of maximal entropy for $\alpha(a)$. Since $\lambda$ is the measure of maximal entropy for $\rho(a)$ then by conjugation so is the measure $\phi_{*}^{-1}(\lambda)$ for $\alpha(a)$. Hence by uniqueness we have $\mu=\phi_{*}^{-1}(\lambda)$.

We will show below that the Lyapunov exponents of $(\alpha, \mu)$ and $(\rho, \lambda)$ are positively proportional and that the corresponding coarse Lyapunov foliations are mapped into each other by the conjugacy $\phi$.

We consider the suspensions $\tilde{\alpha}$ and $\tilde{\rho}$ of $\alpha$ and $\rho$. These are smooth $\mathbb{R}^{k}$ actions on the suspension manifolds $\mathcal{S}$ and $\mathcal{R}$ of $\alpha$ and $\rho$. We denote the lifts to the suspensions of the conjugacy and the invariant measures by $\widetilde{\phi}, \tilde{\mu}$, and $\tilde{\lambda}$. Note that $\widetilde{\phi}$ and $\widetilde{\phi}^{-1}$ are also Hölder continuous with the same exponent $\gamma>0$ as $\phi$ and $\phi^{-1}$.

From now on, instead of indexing a coarse Lyapunov by a representative of the class of positively proportional Lyapunov functionals, we index them numerically. That is, we write $\mathcal{W}^{i}$ instead of $\mathcal{W}^{\chi}$, implicitly identifying the finite collection of equivalence classes of Lyapunov exponents with a finite set of integers. The next proposition summarizes important properties of the suspension actions. Similar properties hold for the original $\mathbb{Z}^{k}$ actions.

Proposition 2.5 Assume there is an Anosov element in every Weyl chamber.

(1) The Lyapunov exponents of $(\tilde{\alpha}, \tilde{\mu})$ and $(\tilde{\rho}, \tilde{\lambda})$ are positively proportional, and thus the Lyapunov hyperplanes and Weyl chambers are the same.

(2) For any coarse Lyapunov foliation $\mathcal{W}_{\widetilde{\alpha}}^{i}$ of $\tilde{\alpha}$

$$
\widetilde{\phi}\left(\mathcal{W}_{\widetilde{\alpha}}^{i}\right)=\mathcal{W}_{\widetilde{\rho}}^{i},
$$

where $\mathcal{W}_{\widetilde{\alpha}}^{i}$ is the corresponding coarse Lyapunov foliation for $\tilde{\rho}$.

Remark This proposition holds for any pair of Hölder conjugate actions satisfying the assumptions of Proposition 2.3. 
Remark One can show that the same conclusions hold for Lyapunov exponents and coarse Lyapunov foliations of $(\alpha, v)$ for any $\alpha$-invariant measure $v$ so, in particular, the Lyapunov exponents of all $\alpha$-invariant measures are positively proportional and the coarse Lyapunov splittings are consistent with the continuous one defined in Proposition 2.3.

Remark We do not claim at this point that the Lyapunov exponents of $(\tilde{\alpha}, \tilde{\mu})$ and $(\tilde{\rho}, \tilde{\lambda})$ (or of different invariant measures for $\widetilde{\alpha}$ ) are equal. Of course if $\widetilde{\alpha}$ is shown to be smoothly conjugate to $\tilde{\rho}$ then this is true a posteriori.

Proof First we observe that the conjugacy $\widetilde{\phi}$ maps the stable manifolds of $\tilde{\alpha}$ to those of $\tilde{\rho}$. More precisely, for any $a \in \mathbb{R}^{k}$ and any for $\mu$-a.e. $x \in \mathcal{S}$ we have

$$
\widetilde{\phi}\left(W_{\widetilde{\alpha}(a)}^{-}(x)\right)=W_{\widetilde{\rho}(a)}^{-}(\widetilde{\phi}(x)) .
$$

Indeed, it suffices to establish this for local manifolds, which are characterized by the exponential contraction as in (2). Since $\widetilde{\phi}$ is bi-Hölder, it preserves the property that $\operatorname{dist}\left(x_{n}, y_{n}\right)$ decays exponentially, which implies (3). In particular, for any Anosov $a \in \mathbb{R}^{k}$ and any $x \in \mathcal{S}$ we have $\widetilde{\phi}\left(W_{\widetilde{\alpha}(a)}^{s}(x)\right)=W_{\widetilde{\rho}(a)}^{s}(\tilde{\phi}(x))$. Hence the formula for $\mathcal{W}_{\widetilde{\alpha}}^{i}$ given in Proposition 2.3 implies (2) once we establish (1).

To establish (1) it suffices to show that the oriented Lyapunov hyperplanes of $(\tilde{\alpha}, \tilde{\mu})$ and $(\tilde{\rho}, \tilde{\lambda})$ are the same. Suppose that an oriented Lyapunov hyperplane $L$ of one action, say $\tilde{\alpha}$, is not an oriented Lyapunov hyperplane of the other action $\tilde{\rho}$. We take an element $a$ close to $L$ in the corresponding positive Lyapunov half-space $L^{+}$and denote the reflection of $a$ across $L$ by $b$. We can choose them so that $a$ and $b$ are not separated by any other Lyapunov hyperplane of either action. Then, $E_{\tilde{\alpha}(b)}^{-}=E_{\tilde{\alpha}(a)}^{-} \oplus E$, where $E$ is the coarse Lyapunov distribution of $\tilde{\alpha}$ corresponding to $L$. Similarly, since we assumed that $L^{+}$is not a positive Lyapunov half-space for $\widetilde{\rho}$, we have $E_{\tilde{\rho}(b)}^{-} \subseteq E_{\tilde{\rho}(a)}^{-}$. We conclude that

$$
W_{\widetilde{\alpha}(a)}^{-} \subsetneq W_{\widetilde{\alpha}(b)}^{-} \text {but } \quad W_{\widetilde{\rho}(a)}^{-} \supseteq W_{\tilde{\rho}(b)}^{-},
$$

which contradicts (3) since $\widetilde{\phi}$ is a homeomorphism.

\section{Outline of the proof of Theorem 1.1}

Proposition 2.3 shows that coarse Lyapunov foliations for $\alpha$ and $\tilde{\alpha}$ are well-defined continuous foliations with smooth leaves. By Proposition 2.5 they are mapped by the conjugacy to the corresponding homogeneous foliations for $\rho$ and $\widetilde{\rho}$. The main goal is to study the conjugacies $\phi$ and $\widetilde{\phi}$ along these foliations. 
For the most of the proof we consider a coarse Lyapunov foliation $\mathcal{W}$ of the suspension action $\tilde{\alpha}$ on $\mathcal{S}$. The first major step is to establish smoothness of certain holonomies between leaves of $\mathcal{W}$. The TNS assumption gives the existence of invariant foliations $\mathcal{W}_{1}$ and $\mathcal{W}_{2}$ such that $T \mathcal{W}_{1} \oplus T \mathcal{W} \oplus T \mathcal{W}_{2} \oplus T \mathcal{O}=T \mathcal{S}$ (see Lemma 5.1). Moreover, each $T \mathcal{W}_{i} \oplus T \mathcal{W}$ is the stable distribution of some element and, in particular, is integrable. In Section 5 we show that the holonomies along $\mathcal{W}_{1}$ (and along $\mathcal{W}_{2}$ ) between leaves of $\mathcal{W}$ are $C^{\infty}$. This follows from the existence of an element for which $\mathcal{W}_{1}$ a fast stable foliation inside $T \mathcal{W}_{1} \oplus T \mathcal{W}$. To obtain such an element we establish in Section 4 that the expansion or contraction of $\mathcal{W}$ by an element in the corresponding Lyapunov hyperplane is uniformly slow.

The second major step is to establish smoothness of the conjugacy $\widetilde{\phi}$ along the leaves of the coarse Lyapunov foliation $\mathcal{W}$. For this we introduce in Section 6 the measurable normal forms for the action on $\mathcal{W}$ defined almost everywhere with respect to the measure $\tilde{\mu}=\tilde{\phi}_{*}^{-1}(\tilde{\lambda})$. In Section 7 we show that the smooth holonomies along $\mathcal{W}_{1}$ preserve the normal forms on $\mathcal{W}$. For this we use the semisimplicity assumption to split the homogeneous foliation $\widetilde{\phi}\left(\mathcal{W}_{1}\right)$ into subfoliations corresponding to eigenspaces of $\rho$. Then we see that holonomies along a particular subfoliation preserve the normal forms since they commute with an element in $\mathbb{R}^{k}$ which fixes the corresponding eigenspace and contracts $\mathcal{W}$. Since $\mathcal{W}_{1}$ is the full stable foliation of some element, it is ergodic with respect to $\mu$, and hence the holonomies along a typical leaf are sufficiently transitive. Using this we show in Section 8 that for a typical leaf $W$ of $\mathcal{W}$ and for almost every translation $T$ of the homogeneous leaf $\widetilde{\phi}(W)$, the conjugate map $\tilde{\phi}^{-1} \circ T \circ \widetilde{\phi}: W \rightarrow W$ can be obtained as a certain limit of such holonomies. Then this map also preserves the normal forms and therefore is smooth. This yields that $\phi$ is $C^{\infty}$ along $W$.

Since the holonomies between different leaves of $\mathcal{W}$ along $\mathcal{W}_{1}$ and $\mathcal{W}_{2}$ are smooth and intertwine the restriction of $\phi$ to these leaves we obtain that $\phi$ is $C^{\infty}$ along all leaves of $\mathcal{W}$ and that the derivatives are continuous transversally. Then standard elliptic theory implies that $\phi$ is $C^{\infty}$ on $M$.

\section{Uniform estimates for elements near Lyapunov hyperplane}

We consider the suspension actions $\tilde{\alpha}$ and $\tilde{\rho}$ of $\mathbb{R}^{k}$ on $\mathcal{S}$ and $\mathcal{R}$. We fix a Lyapunov hyperplane $L \subset \mathbb{R}^{k}$ and the corresponding positive Lyapunov half-space $L^{+}$. We denote the corresponding coarse Lyapunov distributions for $\tilde{\alpha}$ and $\tilde{\rho}$ by $E$ and $\bar{E}$ respectively. Recall that $\gamma>0$ denotes a Hölder exponent of $\widetilde{\phi}$ and $\widetilde{\phi}^{-1}$. 
Lemma 4.1 Consider an element $b \in \mathbb{R}^{k}$. Let $\bar{\chi}(b)$ be the largest Lyapunov exponent of $\tilde{\rho}(b)$ corresponding to $\bar{E}$ and denote $\chi_{M}=\max \{0, \bar{\chi}(b) / \gamma\}$. Let $v$ be any ergodic invariant measure for $\tilde{\alpha}(b)$ and let $\chi_{\nu}(b)$ be the largest Lyapunov exponent of $(\tilde{\alpha}(b), v)$ corresponding to the distribution $E$. Then $\chi_{\nu}(b) \leq \chi_{M}$.

Proof Suppose that $\chi_{v}(b)>\chi_{M}$. Let $E^{u u}$ be the distribution spanned by the Lyapunov subspaces of $(\widetilde{\alpha}(b), v)$ corresponding to Lyapunov exponents greater than $\chi_{M}+\varepsilon$. Then, for some $\varepsilon>0, E^{u u}$ has nonzero intersection with the distribution $E$. The strong unstable distribution $E^{u u}(x)$ is tangent for $v$-a.e. $x$ to the corresponding strong unstable manifold $W^{u u}(x)$. Hence the intersection $F(x)$ of $W^{u u}(x)$ with the leaf $W(x)$ of the coarse Lyapunov foliation corresponding to $E$ is a submanifold of positive dimension. Take $y \in F(x)$ and denote $y_{n}=\tilde{\alpha}(-n b)(y)$ and $x_{n}=\tilde{\alpha}(-n b)(x)$. Then $x_{n}$ and $y_{n}$ converge exponentially with the rate at least $\chi_{M}+\varepsilon$. Since the conjugacy $\tilde{\phi}$ is $\gamma$ bi-Hölder it is easy to see that

$$
\operatorname{dist}\left(\widetilde{\phi}\left(x_{n}\right), \widetilde{\phi}\left(y_{n}\right)\right)=\operatorname{dist}(\widetilde{\rho}(-n b)(x), \tilde{\rho}(-n b)(y))
$$

decreases at a rate faster than $\gamma \chi_{M}$. But this is impossible since $\widetilde{\phi}$ maps $W(x)$ to the corresponding foliation of the linearization which is contracted by $\tilde{\rho}(-b)$ at a rate at most $\gamma \chi_{M}$.

Proposition 4.2 Let $L \subset \mathbb{R}^{k}$ be a Lyapunov hyperplane and $E$ be the corresponding coarse Lyapunov distribution for $\tilde{\alpha}$. For any $\varepsilon>0$ there exist $\eta>0$ so that for any element $b \in \mathbb{R}^{k}$ with $\operatorname{dist}(b, L) \leq \eta \varepsilon$ there exists $C=C(b, \varepsilon)$ such that

$$
\left(C e^{\varepsilon n}\right)^{-1}\|v\| \leq\|D(\widetilde{\alpha}(n b)) v\| \leq C e^{\varepsilon n}\|v\| \quad \text { for all } v \in E, n \in \mathbb{N} .
$$

Proof In the proof we will abbreviate $\tilde{\alpha}(b)$ to $b$. Consider functions $a_{n}(x)=$ $\log \left\|\left.D b^{n}\right|_{E}(x)\right\|, n \in \mathbb{N}$. Since the distribution $E$ is continuous, so are the functions $a_{n}$. The sequence $a_{n}$ is subadditive, ie $a_{n+k}(x) \leq a_{n}\left(b^{k}(x)\right)+a_{k}(x)$. The Subadditive and Multiplicative Ergodic Theorems imply that for every $b$-invariant ergodic measure $v$ the $\operatorname{limit}_{n \rightarrow \infty} a_{n}(x) / n$ exists for $v$-a.e. $x$ and equals the largest Lyapunov exponent of $(b, v)$ on the distribution $E$.

The largest exponent $\bar{\chi}(b)$ of $\tilde{\rho}(b)$ from Lemma 4.1 can be estimated from above by $c \cdot \operatorname{dist}(b, L)$ for some $c>0$. Hence we can find $\eta>0$ so that the number $\chi_{M}$ from Lemma 4.1 is less than $\varepsilon / 2$ for all $b \in \mathbb{R}^{k}$ with $\operatorname{dist}(b, L) \leq \eta \varepsilon$. Then Lemma 4.1 implies that $\lim _{n \rightarrow \infty} a_{n}(x) / n \leq \varepsilon / 2$ for almost every $x$ with respect to any $b-$ invariant ergodic measure $v$. Thus the exponential growth rate of $\left\|\left.D b^{n}\right|_{E}(x)\right\|$ is less than $\varepsilon / 2$ for all $b$-invariant ergodic measures. Since $\left\|\left.D b^{n}\right|_{E}(x)\right\|$ is continuous, this implies the uniform exponential growth estimate, as in the second inequality 
in (4) (see [26, Theorem 1] or [25, Proposition 3.4]). The first inequality in (4) can be obtained from the second one for $-b$.

\section{Smooth holonomies}

We consider the suspension actions $\tilde{\alpha}$ and $\tilde{\rho}$ of $\mathbb{R}^{k}$ on $\mathcal{S}$. We fix a Lyapunov hyperplane $L \subset \mathbb{R}^{k}$ and denote by $E$ and $\mathcal{W}$ the corresponding coarse Lyapunov distribution and foliation for $\tilde{\alpha}$ on $\mathcal{S}$. These are unique by the TNS hypothesis as there are no negatively proportional Lyapunov exponents. In this section we establish smoothness of certain holonomies between leaves of $\mathcal{W}$.

We first need a technical result on existence of suitable complementary foliations.

Lemma 5.1 For a TNS action $\alpha$, suppose that every Weyl chamber contains an Anosov element. Then there are $\tilde{\alpha}$-invariant distributions $E_{1}$ and $E_{2}$ such that $E_{1} \oplus E \oplus E_{2} \oplus T \mathcal{O}=T \mathcal{S}$. Moreover, both $E_{i}$ and $E_{i} \oplus E, i=1,2$ are the stable distribution of some Anosov elements, and hence are tangent to invariant foliations which we denote respectively by $\mathcal{W}_{i}$ and $\mathcal{W}_{i} \oplus \mathcal{W}, i=1,2$.

Proof Consider a generic plane $P$ in $\mathbb{R}^{k}$ which intersects different Lyapunov hyperplanes in distinct lines $L_{i}$. Recall that the TNS assumption implies that each Lyapunov hyperplane bounds a unique negative Lyapunov half-space. Thus the $L_{i}$ are naturally oriented, and we can order them cyclically $L=L_{1}, L_{2}, \ldots, L_{n}$. Let $m$ be the index such that $-L_{1}$ is between $L_{m}$ and $L_{m+1}$. There are two Weyl chambers in the negative Lyapunov half-space $L_{1}^{-}$whose intersections with the plane $P$ border $L_{1}$. By assumption, there exist Anosov elements in these Weyl chambers, which we denote $a_{1}$ and $a_{2}$. Similarly, there are two Weyl chambers across $L_{1}$ in the positive Lyapunov half-space $L_{1}^{+}$. We denote Anosov elements in these Weyl chambers by $c_{1}$ and $c_{2}$. More precisely, if we order the Weyl chambers intersecting $P$ cyclically from $L_{1}: \mathcal{C}_{i}$, $i=1, \ldots, n$ then we can take $a_{1} \in \mathcal{C}_{1}, a_{2} \in \mathcal{C}_{m}, c_{2} \in \mathcal{C}_{m+1}, c_{1} \in \mathcal{C}_{n}$.

Denote the coarse Lyapunov distribution corresponding to $L_{i}$ by $E^{i}$. Note that $E=E^{1}$. Then one can see that $T \mathcal{S}=E_{1} \oplus E \oplus E_{2} \oplus T \mathcal{O}$, where

$$
\begin{array}{ll}
E_{2}:=E_{c_{2}}^{s}=E^{2} \oplus \cdots \oplus E^{m}, & E_{a_{2}}^{s}=E^{1} \oplus \cdots \oplus E^{m}=E \oplus E_{2}, \\
E_{1}:=E_{c_{1}}^{s}=E^{m+1} \oplus \cdots \oplus E^{n}, & E_{a_{1}}^{s}=E^{m+1} \oplus \cdots \oplus E^{n} \oplus E^{1}=E_{1} \oplus E .
\end{array}
$$

Since stable distributions of Anosov elements integrate to invariant foliations, the claim follows. 
We will show that the holonomies along $\mathcal{W}_{i}, i=1,2$ between leaves of $\mathcal{W}$ are $C^{\infty}$. This follows from the existence of an element which contracts $\mathcal{W}_{1}$ (resp. $\mathcal{W}_{2}$ ) faster than it does $\mathcal{W}$.

Proposition 5.2 In the above notation, for $i=1,2$, there exist elements $b_{i} \in \mathbb{R}^{k}$ such that $b_{i}$ contracts $\mathcal{W}_{i}$ faster than it does $\mathcal{W}$, ie

$$
\left\|\left.D\left(\tilde{\alpha}\left(b_{i}\right)\right)\right|_{E_{i}}\right\|<\left\|\left.D\left(\tilde{\alpha}\left(-b_{i}\right)\right)\right|_{E}\right\|^{-1} \leq\left\|\left.D\left(\tilde{\alpha}\left(b_{i}\right)\right)\right|_{E}\right\|<1 .
$$

Since the faster part of an (un)stable foliation is $C^{\infty}$ inside of an (un)stable leaf (see for example [13, Proposition 5.1] or [14, Proposition 3.9]), we obtain the following corollary:

Corollary 5.3 In the above notation, for $i=1,2$, the leaves of $\mathcal{W}_{i}$ vary smoothly along the leaves of $\mathcal{W}$, and the holonomies along $\mathcal{W}_{i}$ between leaves of $\mathcal{W}$ are $C^{\infty}$.

Proof of Proposition 5.2 We use the notation from the proof of Lemma 5.1. We will first find an element $b^{\prime}$ close to $L$ which does not expand or contract $E$ much, with uniform control. Then a suitable combination of $a_{i}$ with $b^{\prime}$ will suffice.

We consider the case $i=1$ and denote $a=a_{1}, c=c_{1}$, and $F=E \oplus E_{1}$. The other cases are similar, and will not be discussed. We have that $a$ uniformly contracts $F$ and $c$ uniformly contracts $E_{1}$, ie there exist $C_{1}, \chi>0$ such that for all $t>0$,

(6) $\|D(\widetilde{\alpha}(t a)) v\| \leq C_{1} e^{-\chi t}\|v\| \quad \forall v \in F, \quad\|D(\widetilde{\alpha}(t c)) v\| \leq C_{1} e^{-\chi t}\|v\| \quad \forall v \in E_{1}$.

Since $E$ is a continuous distribution on $\mathcal{S}$ and $\mathcal{S}$ is compact, $a$ contracts $E$ by at most $\sup _{x \in \mathcal{S}}\left\|\left.d a^{-1}\right|_{E}(x)\right\|$. Hence there is a fastest contraction rate $\chi^{\prime}$ for $a$ on $E$ such that for some $c_{2}>0$ and all $t>0$,

$$
\|D(\widetilde{\alpha}(t a)) v\| \geq c_{2} e^{-\chi^{\prime} t}\|v\| \quad \forall v \in E .
$$

Since $F \supset E$, equations (6) and (7) imply that $\chi^{\prime}>\chi$.

Let $b^{\prime}=r a+(1-r) c, 0<r<1$, be a convex combination of $a$ and $c$. Note that by (6) any such $b^{\prime}$ uniformly contracts $E_{1}$ :

$$
\left\|D\left(\widetilde{\alpha}\left(t b^{\prime}\right)\right) v\right\| \leq C_{1}^{2} e^{-\chi t}\|v\| \quad \forall v \in E_{1}, \forall t>0 .
$$

We will find an element satisfying (5) in the form $b=t\left(b^{\prime}+s a\right)$, where $t>0$ is large and $s>0$ is small. For any $\varepsilon>0$ we can choose $b^{\prime}$ so that it is in $L^{-}$and sufficiently 
close to $L$ so that Proposition 4.2 applies and so $b^{\prime}$ contracts $E$ very slowly. Then equations (6), (7), (8) yield that there exists $K>0$ such that for all $t>0$

$$
\|D(\widetilde{\alpha}(b)) v\| \leq K e^{-(\chi+s \chi) t}\|v\| \quad \forall v \in E_{1},
$$

and

$$
K^{-1} e^{-\left(s \chi^{\prime}+\varepsilon\right) t}\|v\| \leq\|D(\widetilde{\alpha}(b)) v\| \leq K e^{-(s \chi-\varepsilon) t}\|v\| \quad \forall v \in E .
$$

We conclude that $b$ will satisfy (5) for sufficiently large $t$ if we choose $\varepsilon$ and $s$ so that $s \chi^{\prime}+\varepsilon<\chi+s \chi$ while $s \chi-\varepsilon>0$. This is equivalent to

$$
\frac{\varepsilon}{\chi}<s<\frac{\chi-\varepsilon}{\chi^{\prime}-\chi}
$$

and hence we can choose such $s$ if $\varepsilon$ is sufficiently small.

\section{Normal forms}

We consider the suspension action $\tilde{\alpha}$ of $\mathbb{R}^{k}$ on $\mathcal{S}$. We fix a Lyapunov hyperplane $L \subset \mathbb{R}^{k}$ and denote by $E$ and $\mathcal{W}$ the corresponding coarse Lyapunov distribution and foliation for $\tilde{\alpha}$.

In this section we study properties of the action along the leaves of $\mathcal{W}$ and introduce smooth coordinate changes along the leaves of $\mathcal{W}$ with respect to which the elements act as certain polynomials. This method was introduced to the study of local rigidity of higher rank abelian actions by Katok and Spatzier [19] and uses the nonstationary normal forms of smooth contractions developed by and Katok [8] and Guysinsky [7]. In contrast to the case of small perturbations of algebraic actions considered in [19], the action $\tilde{\alpha}$ may not have the so-called "narrow band" property. Instead of uniform growth estimates given by the narrow Mather spectrum, we have to use nonuniform estimates given by the Multiplicative Ergodic Theorem for the measure $\mu$. Therefore, the coordinate changes will vary on $\mathcal{S}$ not continuously but measurably.

Let $a$ be an element in the negative Lyapunov half-space $L^{-} \subset \mathbb{R}^{k}$, so that $f=\widetilde{\alpha}(a)$ contracts $\mathcal{W}$. We will view it as a measure-preserving system $(f, \mu)$. Its action along $\mathcal{W}, f: \mathcal{W}(x) \rightarrow \mathcal{W}(f x)$, defines an extension $\Phi: \mathcal{S} \times \mathbb{R}^{m} \rightarrow \mathcal{S} \times \mathbb{R}^{m}$ of $f$, where $m=\operatorname{dim} \mathcal{W}$. Indeed, the leaf $\mathcal{W}(x)$ can be smoothly identified with the tangent space $E(x)$, and the distribution $E$ can always be measurably trivialized on a set of full measure. The extension $\Phi_{a}$ preserves the zero section and acts by $C^{\infty}$ diffeomorphisms in the fibers. In other words, $\Phi_{a}$ can be written in coordinates $(x, t) \in \mathcal{S} \times \mathbb{R}^{m}$ as

$$
\Phi_{a}(x, t)=\left(f(x), F_{x}(t)\right)
$$

where $F_{x}(0)=0$ and $F$ is $C^{\infty}$ in $t$. We will allow coordinate changes which are measurable in $x$, preserve each fiber $\mathbb{R}_{x}^{m}$, fix the origin, are $C^{\infty}$ in each fiber, and 
have tempered logarithms of all derivatives of all orders at the zero section. We will call such coordinate changes admissible. Recall that a real-valued function $\varphi$ is called tempered with respect to the action $\tilde{\alpha}$ if $\lim _{b \rightarrow \infty}\|b\|^{-1} \varphi(\widetilde{\alpha}(b) x)=0$ for $\mu$-a.e. $x$.

The derivatives in the $t$ variable at the zero section define a linear extension of $f$, which we will denote by $D_{0} F_{X}$ and call the derivative extension. Note that $D_{0} F_{X}$ are bounded functions on $\mathcal{S}$ and that this extension has negative Lyapunov exponents. Let $\chi_{1}, \ldots, \chi_{l}$ be the different Lyapunov exponents of the derivative extension and $m_{1}, \ldots, m_{l}$ be their multiplicities. Represent $\mathbb{R}^{m}$ as the direct sum of the spaces $\mathbb{R}^{m_{i}}, \ldots, \mathbb{R}^{m_{l}}$ and let $\left(t_{1}, \ldots, t_{l}\right)$ be the corresponding coordinate representation of a vector $t \in \mathbb{R}^{m}$. Let $P: \mathbb{R}^{m} \rightarrow \mathbb{R}^{m} ;\left(t_{1}, \ldots, t_{l}\right) \mapsto\left(P_{1}\left(t_{1}, \ldots, t_{l}\right), \ldots, P_{l}\left(t_{1}, \ldots, t_{l}\right)\right)$ be a polynomial map preserving the origin. We will say that the map $P$ is of subresonance type if it contains only such homogeneous terms in $P_{i}\left(t_{1}, \ldots, t_{l}\right)$ with degree of homogeneity $s_{j}$ in the coordinates of $t_{j}, i=1, \ldots, l$ for which the subresonance relation $\chi_{i} \leq \sum_{j \neq i} s_{j} \chi_{j}$ holds. There are only finitely many subresonance relations and it is known $[7 ; 8]$ that polynomial maps of the subresonance type with invertible derivative at the origin generate a finite-dimensional Lie group. We will denote this group by $\mathrm{SR}_{\chi}$.

Proposition 6.1 There exists an admissible coordinate change in $\mathcal{S} \times \mathbb{R}^{m}$ which transforms the extensions $\Phi_{a}$ for all $a \in L^{-}$to extensions $\Psi_{a}$ of the subresonance normal form

$$
\Psi(x, t)=\left(f(x), \mathcal{P}_{x}(t)\right)
$$

where for almost every $x \in X, \mathcal{P}_{x} \in \mathrm{SR}_{\chi}$.

Moreover, this admissible coordinate change transforms into such normal form any extension $\Gamma(x, t)=\left(g(x), \mathcal{G}_{x}(t)\right)$ by $C^{\infty}$ diffeomorphisms preserving the zero section of a nonsingular transformation $g$ of $(\mathcal{S}, \mu)$ which commutes with $\Phi_{a}$ for some $a \in L^{-}$.

Proof We note that since $E$ is a coarse Lyapunov distribution, all Lyapunov exponents of $\tilde{\alpha}$ corresponding to $E$ are, by definition, positively proportional. Therefore, the extensions $\Phi_{a}$ for all $a \in L^{-}$are contractions with the same subresonance relations. The existence of an admissible coordinate change for a single $a^{*} \in L^{-}$is given by Theorem 6.1 in [11]. Since $\Phi_{a}$ commutes with $\Phi_{a^{*}}$, the "centralizer theorem" [11, Theorem 6.3] yields that this coordinate change brings any other $\Phi_{a}$, for $a \in L^{-}$, to the subresonance normal form of $\Phi_{a^{*}}$. The coincidence of resonances implies that this normal form is also the normal form for $\Phi_{a}$. Then the "centralizer theorem" can be applied to this coordinate change with any $a \in L^{-}$and yields the second part of the proposition. 


\section{Commuting holonomies}

Let $\mathcal{W}$ be a coarse Lyapunov foliation as in Section 5 with a complementary foliation $\mathcal{W}_{1}$ as in Lemma 5.1. To simplify notation in Section 7 and Section 8 we will denote the corresponding foliations for the algebraic action $\tilde{\rho}$ by $\mathcal{W}^{*}$ and $\mathcal{W}_{1}^{*}$ respectively. In this section we will study the holonomies along $\mathcal{W}_{1}$ between leaves of $\mathcal{W}$. While in a general setting holonomy along a foliation is only a locally defined operation, in our setting holonomies are realized by global homeomorphisms. Before specifying this we introduce some notation and describe the algebraic foliations $\mathcal{W}^{*}$ and $\mathcal{W}_{1}^{*}$.

We have two actions $\widetilde{\alpha}$ and its linearization $\tilde{\rho}$ on the suspension manifold $\mathcal{S}$. Recall that $\mathcal{S}$ is a homogeneous space $S / \Lambda$ where $S=\mathbb{R}^{k} \ltimes N$ is a solvable Lie group and $\Lambda=\mathbb{Z}^{k} \ltimes \Gamma$ is a lattice in $S$. A left coset foliation is the foliation defined by orbits of some subgroup $D<S$. The foliations $\mathcal{W}^{*}$ and $\mathcal{W}_{1}^{*}$, as well as other coarse Lyapunov and stable foliations for the $\tilde{\rho}$ on $\mathcal{S}$, are left coset foliations. This is most easily seen at the level of Lie algebras. Let $\mathfrak{s}$ be the Lie algebra of $S$ and $\mathfrak{n}$ the Lie algebra of $N$. We can identify the tangent bundle of $\mathcal{S}$ with $\mathcal{S} \times \mathfrak{s}$. The fibration $N / \Gamma \rightarrow \mathcal{S} \rightarrow \mathbb{R}^{k} / \mathbb{Z}^{k}$ of $N / \Gamma$ defines a foliation whose tangent bundle is given by $\mathfrak{n}$ in this identification. Since $\tilde{\rho}$ is the suspension of the action $\rho$ by affine automorphisms of $N / \Gamma$ any "dynamical" foliation as above is tangent to an invariant distribution given by a subspace $\mathfrak{d} \subset \mathfrak{n}$, which by integrability is a Lie subalgebra of $\mathfrak{n}$. This makes the corresponding foliation into a left coset foliation for the subgroup $D<N$ such that Lie $(D)=\mathfrak{d}$. For the coarse Lyapunov foliation $\mathcal{W}^{*}$, and for the complementary foliation $\mathcal{W}_{1}^{*}$ we will denote the corresponding nilpotent groups by $\mathbf{W}$ and $\mathbf{W}_{1}$.

Recall that by Lemma $5.1 \mathcal{W}^{*}$ and $\mathcal{W}_{1}^{*}$ subfoliate the leaves of $\mathcal{W}^{*} \oplus \mathcal{W}_{1}^{*}$, which is a stable foliation for $\tilde{\rho}$. Moreover, on each leaf of this foliation they form a global product structure. This can be seen on the universal cover, which for the algebraic action on $N / \Gamma$ can be identified with the Lie algebra $\mathfrak{n}$. We choose any element $b \in \mathbf{W}_{1}$ and denote the translation action of $b$ on $\mathcal{S}$ by $L_{b}(x)=b \cdot x=b x$. Then for any such $b$ and any $x$ in $\mathcal{S}$ the holonomy along $\mathcal{W}_{1}^{*}$ is a diffeomorphism between $\mathcal{W}^{*}(x)$ and $\mathcal{W}^{*}(b x)$, which we denote by $h_{b, x}^{*}$.

Similarly, for any $b$ in $\mathbf{W}_{1}$ and any $x$ in $\mathcal{S}$ we denote by $h_{b, x}$ the holonomy along $\mathcal{W}_{1}$ between $\mathcal{W}(x)$ and $\mathcal{W}(b x)$. Since the conjugacy $\widetilde{\phi}$ maps $\mathcal{W}$ to $\mathcal{W}^{*}$ and $\mathcal{W}_{1}$ to $\mathcal{W}_{1}^{*}$ we see that $h_{b, x}$ is a global homeomorphism and $h_{b, x}^{*} \circ \widetilde{\phi}=\widetilde{\phi} \circ h_{b, x}$. Moreover, $h_{b, x}$ is a diffeomorphism by Corollary 5.3.

In order to use Proposition 6.1 we can use the holonomies $h_{b, x}^{*}$ and $h_{b, x}$ to define bundle maps in the following manner. We take a bundle $\mathcal{S} \times \mathbf{W}$ with base $\mathcal{S}$ and 
fiber $\mathbf{W}_{s}$, which we think of as the leaf of $\mathcal{W}^{*}$ or $\mathcal{W}$ through $s$. For the leaves of $\mathcal{W}^{*}$ we have a natural identification of $\mathbf{W}_{s}$ with $\mathcal{W}^{*}(s)$ given by left translations: $w \mapsto w \cdot s$. For $\mathcal{W}$ we fix some smooth identification that depends continuously on $s$ in $C^{\infty}$ topology on compact subsets of $\mathbf{W}$. The holonomy $h_{b}^{*}$ can now be viewed as a bundle map $h_{b}^{*}: \mathcal{S} \times \mathbf{W} \rightarrow \mathcal{S} \times \mathbf{W}$ covering the left translation $L_{b}$ in the base, where $h_{b}^{*}(x, w)=h_{b, x}^{*}(w)$.

Similarly, we define $h_{b}$ via the equation $h_{b}(x, w)=h_{b, x}(w)$ and $h_{b}$ is a bundle map $h_{b}: \mathcal{S} \times \mathbf{W} \rightarrow \mathcal{S} \times \mathbf{W}$ which is smooth along the fibers and covers the homeomorphism $\widetilde{\phi}^{-1} \circ L_{b} \circ \widetilde{\phi}$ of $\mathcal{S}$. Note that this homeomorphism preserves the invariant measure $\mu$ on $\mathcal{S}$ since $L_{b}$ preserves the Lebesgue measure $\lambda=\widetilde{\phi}_{*}(\mu)$. Since the nilpotent group $\mathbf{W}$ is diffeomorphic to $\mathbb{R}^{m}$, we are in the setup of Proposition 6.1. The actions $\tilde{\alpha}$ and $\tilde{\rho}$ also lift naturally to the corresponding actions on the bundle $\mathcal{S} \times \mathbf{W}$. Slightly abusing notation we will denote the lifts by the same letters. Since we do not know the smoothness of $\mathcal{W}$, we can only say that the lift of $\tilde{\alpha}$ is smooth along the fibers. Note that the natural extension of $\widetilde{\phi}$ to $\mathcal{S} \times \mathbf{W}$ conjugates the lifts of the actions as well as the holonomy maps $h_{b}$ and $h_{b}^{*}$.

We will use the algebraic structure of $\tilde{\rho}$ to show that $h_{b}^{*}$ commute with certain elements of $\tilde{\rho}\left(\mathbb{R}^{k}\right)$ and then use this to conclude that $h_{b}$ commute with certain elements of $\widetilde{\alpha}\left(\mathbb{R}^{k}\right)$. The main goal of this section is to prove the following theorem.

Theorem 7.1 If the action $\rho$ is semisimple, then for every $b \in \mathbf{W}_{1}$ the maps $h_{b}$ for all $b \in \mathbf{W}$ preserve, $\mu$-almost everywhere, a fixed normal form along leaves of $\mathcal{W}$.

Proof We begin by finding subfoliations of $\mathcal{W}_{1}$ for which the holonomy commutes with some $\tilde{\alpha}(v), v \in \mathbb{R}^{k}$, contracting $\mathcal{W}$. To do this we will work with the algebraic action $\tilde{\rho}$. Recall that $T \mathcal{W}^{*}$ splits as a sum of coarse Lyapunov distributions $\bigoplus E^{j}$. Let $E^{\prime}$ be one of these distributions and $L^{\prime}$ be the corresponding Lyapunov hyperplane. Let $v$ be any element of $L^{\prime}$ for which $\tilde{\rho}(v)$ contracts $\mathcal{W}^{*}$. Then $\widetilde{\rho}(v)$ acts isometrically on a certain foliation $\mathcal{H}_{v}^{*}$ which is defined as the orbits of the action of some subgroup $\mathbf{H}_{v}$ in $\mathbf{W}_{1}$. Since $\tilde{\rho}(v)$ is semisimple by the assumption, $\mathcal{H}_{v}^{*}$ is in fact the full coarse Lyapunov foliation of $\tilde{\rho}$ corresponding to $L^{\prime}$. (If the derivative of $\tilde{\rho}(v)$ on $E^{\prime}$ had Jordan blocks, this would be a strict subfoliation.) Now we can decompose the Lie algebra of $\mathbf{H}_{v}$ into the irreducible subspaces of the rotation defined by taking the skew symmetric part of $\tilde{\rho}(v)$. We denote the resulting Lie subgroups of $\mathbf{H}_{v}$ by $\mathbf{H}_{v, i}$.

Lemma 7.2 For any element $v \in L^{\prime}$ there are real numbers $t_{i}>0$ such that for any $b \in \mathbf{H}_{v, i}$ the map $h_{b}^{*}$ commutes with $\tilde{\rho}\left(t_{i} v\right)$. 
Proof A suitable multiple $t_{i} v$ of $v$ commutes with the group $\mathbf{H}_{v, i}$. Hence translations by elements of $\mathbf{H}_{v, i}$ commute with $\tilde{\rho}\left(t_{i} v\right)$. Then the holonomy $h_{b}^{*}$ will also commute with $\widetilde{\rho}\left(t_{i} v\right)$ since it agrees with holonomy along the foliation $\mathcal{H}_{v, i}^{*}$.

Since $\widetilde{\phi}$ conjugates the actions and the holonomies this lemma yields that for any $b \in \mathbf{H}_{v, i}$ the map $h_{b}$ commutes with $\tilde{\alpha}\left(t_{i} v\right)$. By making different choices of the Lyapunov hyperplane $L^{\prime}, v \in L^{\prime}$, and $i$, we can arrange so that the groups $\mathbf{H}_{v, i}$ generate $\mathbf{W}_{1}$. Proposition 6.1 implies that there is a common normal form for $h_{b}$ for all $b$ in all $\mathbf{H}_{v, i}$. Therefore, $h_{b}$ with any $b$ in $\mathbf{W}_{1}$, which is a composition of maps of this form, also preserves this normal form. This completes the proof of Theorem 7.1.

Now we give a more detailed description of the algebraic holonomies $h_{b}^{*}$. As a corollary we describe certain limits of the maps $h_{b}^{*}$ and $h_{b}$ which will be used in the next section.

Proposition 7.3 For any $b \in \mathbf{W}_{1}$ and any $x \in \mathcal{S}$, the holonomy $h_{b, x}^{*}: W^{*}(x) \rightarrow$ $W^{*}(b x)$ is equivariant with respect to the action of $\mathbf{W}$ along leaves of $\mathcal{W}^{*}$.

Proof We need to show that the holonomy along $\mathcal{W}_{1}^{*}$ commutes with the action of $\mathbf{W}$ along leaves of $\mathcal{W}^{*}$. First we observe that $\mathbf{W}$ normalizes $\mathbf{W}_{1}$. To see this we note that there is a subgroup $\mathbf{W}^{\prime}=\mathbf{W W}_{1}$ in $N$. This is the group that corresponds to the foliation $\mathcal{W}^{*} \oplus \mathcal{W}_{1}^{*}$ as in Lemma 5.1. We denote the Lie algebras of $\mathbf{W}$ and $\mathbf{W}_{1}$ by $\mathfrak{w}$ and $\mathfrak{w}_{1}$. To conclude that $\mathbf{W}_{1}$ is normal inside $\mathbf{W}^{\prime}$ we choose an element $s \in \mathbb{R}^{k}$ for which $\tilde{\rho}(s)$ acts isometrically on $\mathfrak{w}$ and contracts exactly $\mathfrak{w}_{1}$. This is possible by the construction of $\mathcal{W}^{*}$ and $\mathcal{W}_{1}^{*}$ in Lemma 5.1. Then any bracket $[v, u]$ where $u \in \mathfrak{w}$ and $v \in \mathfrak{w}_{1}$ is contracted by $\tilde{\rho}(s)$ and hence must be in $\mathfrak{w}_{1}$.

Now let $a \in \mathbf{W}$ and $b \in \mathbf{W}_{1}$. For any $x \in \mathcal{S}$ we can write $a b x=a b a^{-1} a x$. By the normalization we have $a b a^{-1} \in \mathbf{W}_{1}$. Hence the point $a b x$ is both in $\mathcal{W}^{*}(b x)$ and in $\mathcal{W}_{1}^{*}(a x)$. This shows that $h_{b, x}^{*}(a x)=a b x$ for any $x \in \mathcal{S}$ and $b \in \mathbf{W}_{1}$ and proves that the holonomy $h_{b, x}^{*}$ from $\mathcal{W}^{*}(x)$ to $\mathcal{W}^{*}(b x)$ commutes with the action of $\mathbf{W}$.

Corollary 7.4 Suppose that for some elements $b_{n} \in \mathbf{W}_{1}$ and some point $x \in \mathcal{S}$ the sequence $b_{n} x$ converges to a point $y$ in $\mathcal{W}^{*}(x)$. Then the holonomy maps $h_{b_{n}, x}^{*}$ converge to the diffeomorphism $T_{x, y}^{*}: \mathcal{W}^{*}(x) \rightarrow \mathcal{W}^{*}(x)$ given by $T_{x, y}^{*}($ ax $)=$ ay for any $a \in \mathbf{W}$. Consequently, the holonomy maps $h_{b_{n}, x}$ converge to the homeomorphism $\tilde{\phi}^{-1} \circ T_{x, y}^{*} \circ \tilde{\phi}$ of the leaf $\mathcal{W}\left(\tilde{\phi}^{-1}(x)\right)$ uniformly on compact sets.

Proof By Proposition 7.3, if $a \in \mathbf{W}$ then $h_{b_{n}, x}^{*}(a x)=a h_{b_{n}, x}^{*}(x)=a\left(b_{n} x\right)$, which converges to $a y$ as desired. The first claim now follows since $\mathcal{W}^{*}(x)=\mathbf{W} x$. Conjugating by $\tilde{\phi}$ gives the second claim.

Remark It is not clear that the limit of the $h_{b_{n}, x}^{*}$ can be realized as a holonomy of any kind along any leaf from $W^{*}(x)$ to $W^{*}(y)$. 


\section{Limiting argument}

The main goal of this section is to prove the following proposition, which we will then use to complete the proof of Theorem 1.1. We retain the notation of the previous section.

Proposition 8.1 For $\mu$-almost every $x \in \mathcal{S}$ there are smooth transitive actions of $\mathbf{W}$ on the leaves $\mathcal{W}(x)$ and $\mathcal{W}^{*}(x)$ which are intertwined by the conjugacy $\tilde{\phi}$.

Proof For any given $x \in \mathcal{S}$ we can naturally identify $\mathbf{W}$ with $\mathcal{W}^{*}(x)=\mathbf{W} x$ by $w \mapsto w x$. In this identification, we take the desired transitive action of $\mathbf{W}$ on $\mathcal{W}^{*}(x)$ to be the action by right translations. Corollary 7.4 means that the limits of the holonomy maps $h_{b_{n}, x}^{*}$ are exactly of this form. In fact, since $\mathcal{W}_{1}^{*}(x)$ is dense in the corresponding $N / \Gamma$-fiber of the suspension as a full stable foliation of Anosov element, one can see that any right translation can be obtained as such a limit. While we will not use it directly, this provides motivation for the argument. The desired action of $\mathbf{W}$ on $\mathcal{W}^{*}(x)$ is obtained by conjugating by $\tilde{\phi}$. This action is a priori only by homeomorphisms and the goal is to prove that it is smooth. For this we will study the limits of the holonomy maps $h_{b_{n}, x}$.

We consider Lusin sets where the measurable normal form on the leaf $\mathcal{W}(x)$ depends continuously on $x$. Let $\Lambda_{m}^{\prime}$ be an increasing sequence of such sets with $\mu\left(\Lambda_{m}^{\prime}\right) \rightarrow 1$. Let $\Lambda_{m}$ be the set of density points of $\Lambda_{m}^{\prime}$, then $\mu\left(\Lambda_{m}\right) \rightarrow 1$. Then there exists a subset $X \subset \Lambda=\cup \Lambda_{m}$ with $\mu(X)=1$ such that for all $x \in X$ the intersection $\mathcal{W}(x) \cap \Lambda$ has full measure with respect to the conditional measure of $\mu$ on $\mathcal{W}(x)$.

Fix any $x \in X$. Then for almost every $y$ in $\mathcal{W}(x)$ with respect to the conditional measure $x$ and $y$ belong to some $\Lambda_{m}$. We pick a sequence $b_{n}$ of elements in $\mathbf{W}_{1}$ with the following properties:

(1) $x_{n}=b_{n} x \rightarrow y$.

(2) $x_{n} \in \Lambda_{m}$.

To find such $b_{n}$ we use the fact that $y$ is a density point of $\Lambda_{m}$ and the fact that $\mathbf{W}_{1}$ acts ergodically with respect to $\mu$ on the corresponding $N / \Gamma$-fiber of the suspension. The ergodicity follows since the foliation $\mathcal{W}_{1}$ of $N / \Gamma$ is a full stable foliation of some Anosov element of $\alpha$ and hence is uniquely ergodic by Bowen and Marcus [3]. Alternately, since the push forward of $\mu$ by $\phi$ is Lebesgue, the ergodicity can be checked on the algebraic side using the work of Auslander, Hahn, and Green [1].

Each map $h_{b_{n}, x}$ is smooth and preserves the normal forms at $x$ and $x_{n}$. By Corollary 7.4 the sequence $h_{b_{n}, x}$ converges to a homeomorphism $T_{x, y}: \mathcal{W} \rightarrow \mathcal{W}$ conjugate 
by $\widetilde{\phi}$ to the translation $T_{\tilde{\phi}(x), \tilde{\phi}(y)}^{*}$ of $\mathcal{W}^{*}(\widetilde{\phi}(x))$. Since the normal form coordinates depend continuously on the base point in $\Lambda_{m}$ and the maps $h_{b_{n}, x}$ in these coordinates belong to a fixed Lie group, the limit $T_{x, y}$ is smooth. Recall that the push forward of $\mu$ by $\tilde{\phi}$ is the Lebesgue measure $\lambda$ and hence the conditional measure of $\mu$ on $\mathcal{W}(x)$ is mapped by $\widetilde{\phi}$ to the conditional measure of $\lambda$ on $\mathcal{W}^{*}(\widetilde{\phi}(x))$, which is equivalent to volume on $\mathcal{W}^{*}(\widetilde{\phi}(x))=\mathbf{W} \widetilde{\phi}(x)$. We conclude that for almost every element of $\mathbf{W}$ the corresponding translation is conjugate by $\widetilde{\phi}$ to a diffeomorphism of $\mathcal{W}(x)$. Hence the subgroup of $\mathbf{W}$ that acts by diffeomorphisms of $\mathcal{W}(x)$ has full measure and must be the whole $\mathbf{W}$ by the next lemma. It now follows from [23, Section 5.1, Corollary] that the action of $\mathbf{W}$ on $\mathcal{W}(x)$ is smooth. This completes the proof of Proposition 8.1.

Lemma 8.2 Let $G$ be a Lie group. Then any subgroup $H$ of full measure is $G$.

Proof If not then the distinct cosets of $H$ in $G$ are disjoint sets of full measure which is impossible.

Remark It is possible to prove that $G$ is smooth along a generic leaf of $\mathcal{W}$ using older methods involving returns along Weyl chamber walls in $\mathbb{R}^{k}$ instead of holonomies. However, one cannot obtain uniformity in estimates this way nor complete the proof below without using holonomies.

End of Proof of Theorem 1.1 We need to show that $\phi$ is a diffeomorphism. It will be easier to work with $\phi^{-1}$ as we will employ certain elliptic operators defined by right invariant vector fields to prove smoothness of $\phi^{-1}$.

Proposition 8.1 implies that for any coarse Lyapunov foliation $\widetilde{\phi}^{-1}$ intertwines transitive $C^{\infty}$ group actions on typical leaves $\mathcal{W}(x)$ and $\mathcal{W}^{*}(x)$ in the suspension $\mathcal{S}$. This yields that, for a typical $x$ in $M=N / \Gamma, \phi^{-1}$ intertwines transitive $C^{\infty}$ group actions on $\mathcal{W}(x)$ and $\mathcal{W}^{*}(x)$. Hence $\phi^{-1}$ is $C^{\infty}$ along $\mathcal{W}^{*}(x)$.

We claim that $\phi^{-1}$ is $C^{\infty}$ along all leaves of $\mathcal{W}^{*}$ and that all its derivatives along the leaves are continuous on $M$. This follows from the fact that $T M=T \mathcal{W} \oplus T \mathcal{W}_{1} \oplus T \mathcal{W}_{2}$ and that the holonomies between different leaves of $\mathcal{W}$ along $\mathcal{W}_{1}$ and $\mathcal{W}_{2}$ are smooth and intertwine the restriction of $\phi^{-1}$ to these leaves.

We can now finish the proof quickly. We know that $\phi^{-1}$ is smooth along the coarse Lyapunov foliations with continuous dependence of the derivatives. This simply says that derivatives of all orders exist and are continuous for each right invariant vector field tangent to a coarse Lyapunov foliations (while mixed derivatives may fail to exist). Pick a basis of such vector fields $X_{i}$. Then $X^{l}:=\sum_{i} X_{i}^{2 l}$ for any $l$ is an elliptic operator of order 21. It follows that $X^{l}\left(\phi^{-1}\right)$ is smooth for all $l$. Hence by elliptic 
theory, $\phi^{-1}$ is $C^{\infty}$. We refer to [5, Section 7.1] eg for a more detailed discussion of this elliptic theory argument.

It remains to show that $\phi^{-1}$ is a diffeomorphism. Since $\phi^{-1}$ is already a homeomorphism, this follows once we show that the differential of $\phi^{-1}$ is everywhere nondegenerate. This follows easily from Proposition 2.4. Indeed, we have $\mu=\phi_{*}^{-1}(\lambda)$ and $\mu$ has smooth positive density.

\section{Totally reducible actions and examples}

Here we will prove Corollary 1.2. By the proposition below, this is immediate from Theorem 1.1.

Recall that an algebraic $\mathbb{Z}^{k}$ action on a torus is called irreducible if there is no rational invariant subtorus, and totally reducible if every rational invariant subtorus has a rational invariant complement.

Given a nilmanifold $N / \Gamma$, there is a maximal toral quotient $\mathbb{T}^{d}$ obtained by taking $N /[N, N] \Gamma$. Any action by automorphisms on $N / \Gamma$ descends to an action on $\mathbb{T}^{d}$, which we refer to as the maximal toral quotient action. We say that an algebraic $\mathbb{Z}^{k}$ action on $N / \Gamma$ is totally reducible if the maximal toral quotient action is totally reducible and there is a $\mathbb{Z}^{k}$ invariant complement to $[\mathfrak{n}, \mathfrak{n}]$ in the Lie algebra $\mathfrak{n}$ of $N$.

We call an action by affine automorphisms of a nilmanifold totally reducible if the finite index subgroup that acts by automorphisms is totally reducible.

It is easy to see that semisimple actions are totally reducible.

Proposition 9.1 A totally reducible $\mathbb{Z}^{k}$ action on a nilmanifold is semisimple.

Proof First we consider an irreducible torus action. Let $A$ be a toral automorphism, ie an integral matrix. The characteristic polynomial of $A$ splits over $\mathbb{Q}$ as $\prod P_{i}(X)^{d_{i}}$. Then the kernel $E(A)$ of $\prod P_{i}(A)$ is the subspace spanned by the eigenspaces of $A$. It is rational as the kernel of a rational operator.

If a collection $A_{i}$ of toral automorphisms commute then $E\left(A_{1}\right)$ is invariant under $A_{2}$. Consider the restriction $B_{2}$ of $A_{2}$ to $E\left(A_{1}\right)$ Then $E\left(B_{2}\right)$ is nontrivial, and contained in $E\left(A_{1}\right) \cap E\left(A_{2}\right)$. Inductively we see that $\bigcap E\left(A_{i}\right)$ is nontrivial. Thus we get a nontrivial rational subspace invariant under all $A_{i}$. This defines an invariant proper subtorus unless all $A_{i}$ are semisimple. Hence irreducible torus actions are semisimple.

Considering irreducible components of totally reducible torus actions it follows easily that they are also semisimple. 
Finally consider a totally reducible action on a nilmanifold. Then the maximal toral quotient action is totally reducible and hence semisimple. This implies that the action on the invariant complement $\mathbb{R}^{d}$ to $[\mathfrak{n}, \mathfrak{n}]$ is semisimple. Since joint eigenvectors for $\mathbb{Z}^{k}$ span $\mathbb{R}^{d}$, their brackets, which are also eigenvectors span $\mathfrak{n}$. Therefore the action is semisimple.

We briefly describe many examples of totally irreducible Anosov actions on nilmanifolds. These examples are more general variants of examples constructed by Qian in [24]. Let $\mathbb{T}^{d}$ be a torus with an Anosov algebraic semisimple $\mathbb{Z}^{k}$ action. The action lifts to the vector space $\mathbb{R}^{d}$. Let $N=N^{k}\left(\mathbb{R}^{d}\right)$ be the $k$-step free nilpotent Lie group generated by $\mathbb{R}^{d}$. (It is somewhat more typical to define this at the level of Lie algebras, but the meaning is clear as long as we assume $N^{k}\left(\mathbb{R}^{d}\right)$ is simply connected.) The $\mathbb{Z}^{k}$ action on $\mathbb{R}^{d}$ extends canonically to a $\mathbb{Z}^{k}$ action on $N^{k}\left(\mathbb{R}^{d}\right)$ and preserves the obvious rational structure on that group. This implies that we have a well-defined $\mathbb{Z}^{k}$ action on $N / \Gamma$ where $\Gamma$ is a lattice in $N$.

It is easy to check that generically this construction takes an Anosov $\mathbb{Z}^{k}$ action on $\mathbb{T}^{d}$ and lifts it to an Anosov action on $N / \Gamma$. An Anosov automorphism $A$ of $\mathbb{T}^{d}$ lifts to an Anosov automorphism of $N / \Gamma$ as long as no product of length at most $k$ of eigenvalues of $A$ has modulus one. It is straightforward to construct many examples which are also TNS using similar algebraic condition on eigenvalues.

We remark that the hypothesis of Theorem 1.1 are necessary for our argument as there are examples for which the commuting holonomies are not ergodic.

Example 9.1 Take a semisimple Anosov linear action of $\mathbb{Z}^{k}$ on $\mathbb{T}^{d}$, we can define an action on $\mathbb{T}^{2 d}$ by letting $A \in \mathbb{Z}^{k}$ act by $A(x, y)=(A x, A y+x)$. It is straightforward to check that for examples of this kind, the commuting holonomies are not ergodic.

\section{References}

[1] L Auslander, L Green, F Hahn, Flows on homogeneous spaces, Annals of Math. Studies 53, Princeton Univ. Press (1963) MR0167569 With the assistance of L Markus and W Massey, and an appendix by L Greenberg

[2] L Barreira, Y B Pesin, Lyapunov exponents and smooth ergodic theory, Univ. Lecture Series 23, Amer. Math. Soc. (2002) MR1862379

[3] R Bowen, B Marcus, Unique ergodicity for horocycle foliations, Israel J. Math. 26 (1977) 43-67 MR0451307

[4] M Einsiedler, T Fisher, Differentiable rigidity for hyperbolic toral actions, Israel J. Math. 157 (2007) 347-377 MR2342454 
[5] D Fisher, G Margulis, Local rigidity of affine actions of higher rank groups and lattices, Ann. of Math. (2) 170 (2009) 67-122 MR2521112

[6] J Franks, Anosov diffeomorphisms on tori, Trans. Amer. Math. Soc. 145 (1969) 117124 MR0253352

[7] M Guysinsky, The theory of non-stationary normal forms, Ergodic Theory Dynam. Systems 22 (2002) 845-862 MR1908557

[8] M Guysinsky, A Katok, Normal forms and invariant geometric structures for dynamical systems with invariant contracting foliations, Math. Res. Lett. 5 (1998) 149-163 MR1618331

[9] M W Hirsch, C C Pugh, M Shub, Invariant manifolds, Lecture Notes in Math. 583, Springer, Berlin (1977) MR0501173

[10] S Hurder, Rigidity for Anosov actions of higher rank lattices, Ann. of Math. (2) 135 (1992) 361-410 MR1154597

[11] B Kalinin, A Katok, Invariant measures for actions of higher rank abelian groups, from: "Smooth ergodic theory and its applications (Seattle, WA, 1999)", (A Katok, R de la Llave, Y Pesin, H Weiss, editors), Proc. Sympos. Pure Math. 69, Amer. Math. Soc. (2001) 593-637 MR1858547

[12] B Kalinin, A Katok, Measure rigidity beyond uniform hyperbolicity: invariant measures for Cartan actions on tori, J. Mod. Dyn. 1 (2007) 123-146 MR2261075

[13] B Kalinin, V Sadovskaya, Global rigidity for totally nonsymplectic Anosov $\mathbb{Z}^{k}$ actions, Geom. Topol. 10 (2006) 929-954 MR2240907

[14] B Kalinin, V Sadovskaya, On the classification of resonance-free Anosov $\mathbb{Z}^{k}$ actions, Michigan Math. J. 55 (2007) 651-670 MR2372620

[15] B Kalinin, R Spatzier, On the classification of Cartan actions, Geom. Funct. Anal. 17 (2007) 468-490 MR2322492

[16] A Katok, B Hasselblatt, Introduction to the modern theory of dynamical systems, Encyc. of Math. and its Appl. 54, Cambridge Univ. Press (1995) MR1326374 With a supplementary chapter by Katok and L Mendoza

[17] A Katok, J Lewis, Local rigidity for certain groups of toral automorphisms, Israel J. Math. 75 (1991) 203-241 MR1164591

[18] A Katok, R Spatzier, First cohomology of Anosov actions of higher rank abelian groups and applications to rigidity, Inst. Hautes Études Sci. Publ. Math. (1994) 131156 MR1307298

[19] A Katok, R Spatzier, Differential rigidity of Anosov actions of higher rank abelian groups and algebraic lattice actions, Tr. Mat. Inst. Steklova 216 (1997) 292-319 MR1632177 
[20] R de la Llave, J M Marco, R Moriyón, Canonical perturbation theory of Anosov systems and regularity results for the Livšic cohomology equation, Ann. of Math. (2) 123 (1986) 537-611 MR840722

[21] A Manning, There are no new Anosov diffeomorphisms on tori, Amer. J. Math. 96 (1974) 422-429 MR0358865

[22] G A Margulis, N Qian, Rigidity of weakly hyperbolic actions of higher real rank semisimple Lie groups and their lattices, Ergodic Theory Dynam. Systems 21 (2001) 121-164 MR1826664

[23] D Montgomery, L Zippin, Topological transformation groups, Robert E Krieger Publishing, Huntington, NY (1974) MR0379739 Reprint of the 1955 original

[24] N T Qian, Anosov automorphisms for nilmanifolds and rigidity of group actions, Ergodic Theory Dynam. Systems 15 (1995) 341-359 MR1332408

[25] F Rodriguez Hertz, Global rigidity of certain abelian actions by toral automorphisms, J. Mod. Dyn. 1 (2007) 425-442 MR2318497

[26] S J Schreiber, On growth rates of subadditive functions for semiflows, J. Differential Equations 148 (1998) 334-350 MR1643183

[27] P Walters, Conjugacy properties of affine transformations of nilmanifolds, Math. Systems Theory 4 (1970) 327-333 MR0414830

Department of Mathematics, Indiana University

Rawles Hall, Bloomington IN 47405, USA

Department of Mathematics and Statistics, University of South Alabama

Mobile AL 36688, USA

Department of Mathematics, University of Michigan

2074 East Hall, 530 Church Street, Ann Arbor MI 48109, USA

fisherdm@indiana.edu, kalinin@jaguar1.usouthal.edu, spatzier@umich.edu

http://mypage.iu.edu/ fisherdm/, http://www.southalabama.edu/mathstat/ personal_pages/kalinin/, http://www.math.lsa.umich.edu/ spatzier/

Proposed: Benson Farb

Seconded: Danny Calegari, Walter Neumann
Received: 29 March 2010 Accepted: 15 November 2010 